\title{
MEASUREMENT AND PREDICTION OF STRESS-STRAIN FOR EXTRUDED OILSEED USING NEURAL NETWORKS UNDER UNIAXIAL COLD PRESSING
}

\author{
Xiao Zheng ${ }^{1, *}$, Guoxiang Lin ${ }^{1}$, Dongping $\mathrm{He}^{2}$, Jingzhou Wang ${ }^{1}$ \\ ${ }^{I}$ Department of Mechanical Engineering, Wuhan Polytechnic University, Wuhan, Hubei \\ Province, P. R. China, 430023 \\ 2 Department of Food Science and Engineering, Wuhan Polytechnic University, Wuhan, \\ Hubei Province, P. R. China, 430023 \\ * Corresponding author, Address: Department of Mechanical Engineering, Wuhan \\ Polytechnic University, Wuhan 430023, Hubei Province, P. R. China, Tel: +86-27- \\ 83956425, Fax: +86-27-83956425, Email: zhengxiao580405@163.com
}

Abstract: A visualization of testing apparatus was developed to measure property of oilseeds relevant to physical mechanics during mechanical pressing for oil extraction. Stress-strain relationships were measured for extruded peanut, soybean, sesame and linseed compressed at thirteen pressures under uniaxial cold pressing. The prediction model of the stress-strain relationship was developed based on BP neural network. Results indicated that the stress-strain relationships were nonlinear. Over 50\% strains for extruded soybean, sesame and linseed occurred at stress below 20MPa. Over $60 \%$ strain for extruded peanut occurred at stress below $10 \mathrm{MPa}$. No more than $13 \%$ strain occurred at stress over $20 \mathrm{MPa}$ for extruded soybean sesame and linseed, and no more than $13 \%$ strain occurred at stress over $10 \mathrm{MPa}$ for extruded peanut. The maximum error between prediction and measurement for the stress-strain relationship was less than 0.0084 and the maximum training times was less than 88 .

Keywords: measurement, prediction, stress-strain, neural networks, oilseed, cold pressing

\section{INTRODUCTION}

Peanut, soybean and sesame oil are important edible oil in the world. The mechanical pressing is the most common method for oil extraction in the world. 
Vegetable oilseed expresses complex mechanics behavior during pressing (Mrema et al., 1985). The conventional method of oil extraction suggests that oilseeds must be thermal (cooking) pretreatments before pressing, which is called the hot pressing (Rasehom et al., 2000; Bargale et al., 1999, 2000). More recently, the cold pressing for oil extraction, which needn't be cooked prior to pressing, is very popular in China as well as in other many countries. The main reason for popularity of the cold pressing is that the cold pressings yields limpid color and fruity oilseed oil with lower phosphorus and fatty acid (Rasehom et al., 2000; Zheng Xiao et al., 2004). However, compared to the hot pressing, the cold pressing is inefficient with lower throughputs and higher residual oil contents in the defiled cake. It indicates that the oil press used to the hot pressing needs further improve for the purpose of the cold pressing (Rasehom et al., 2000; Zheng Xiao et al., 2004).

The stress-strain relationship is the most important performance of physical mechanics for extruded oilseeds. The stress-strain model for extruded oilseeds by cold pressing is essential to rigorous theoretical analysis of mechanisms and physical processes. It lays a foundation for problems of permeability, differential equation for seepage (Zheng Xiao et al., 2004). Davison et al. $(1975,1979)$ have studied mechanical properties of single rapeseeds. Sukumaran et al. (1989) have studied bulk properties of rapeseeds under compression. However, the research relating to stress-strain relationship for extruding oilseed has not yet been reported in the world up till now except research for rapeseed and dehulled rapeseed (Zheng Xiao et al., 2004).

It is found very difficult to develop the theoretical model for stress-strain relationship for oilseeds due to the complexity of physical mechanics performance during pressing (Zheng Xiao et al., 2004). At present, multivariable nonlinear regression analysis is most common method to develop empirical formula to predict stress-strain relationship for complex material. However, the difference in the variable used in the analytical model and the details of the experiment will lead to significant diversity in the calculation formulas, and furthermore there is usual a difficulty to determine suitable regression equation used in multiple regression analysis, which requires considerable technique and experience due to understanding of the data characteristic of stress-strain experiment. The objectives of this study were to measure stress-strain for extruding oilseeds by uniaxial cold pressing, and develop neural network modeling to predict the stress-strain relationship. 


\section{STRESS-STRAIN EXPERIMENT}

\subsection{Design of visualized compression cell}

A visualization of test apparatus used for the experiment was specially designed. Its schematic diagram is shown in Fig. 1. It mainly consists of a loading piston, an outer cylinder, an inner cylinder, a sealing ring, a support plate, a porous stone and a base plate. The test apparatus is mounted in a universal hydraulic test machine capable of applying compressive loads of $300 \mathrm{KN}$. The pressing chamber is provided with a $44 \mathrm{~mm}$ diameter $\times 95 \mathrm{~mm}$ deep bore through which the loading piston compresses sample. The visual cylinder is made of plexiglas. An outer cylinder made of mild steel is essential to visual cylinder in order to increase its strength and rigidity. The outer cylinder is provided with two observed windows with a $20 \mathrm{~mm}$ width $\times$ $25 \mathrm{~mm}$ height. The performance and phenomenon of compressive process of oilseed samples can be observed through the visual inner cylinder. Support plate made of stainless-steel with several $3 \mathrm{~mm}$ diameter traverse holes distributed uniformly is designed to prevent porous stone from breaking. In order to ensure uniform fluid pressure within oilseed cakes, both the bottom of loading piston and the top of base plate are provided with radial and circular grooves $5 \mathrm{~mm}$ width $\times 5 \mathrm{~mm}$ depth. The top and bottom of oilseed sample are respectively provided with a porous stone in order to expel liquid (including oil and water) and air from oilseed during compression.

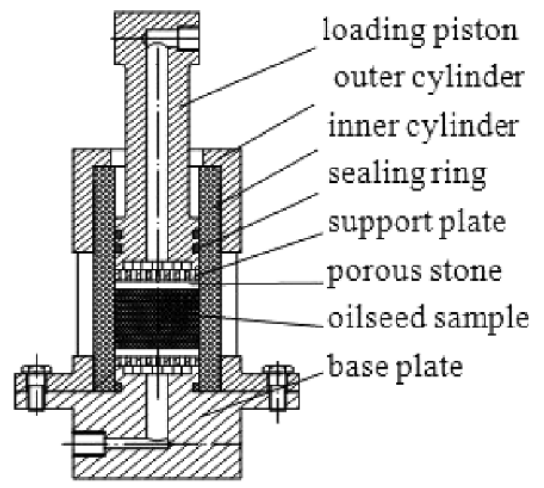

Fig. 1. Schematic diagram of visualized compression cell

\subsection{Measurement of stress-strain}

A 30g sample was chosen as testing specimen for the experiment of peanut, soybean, sesame and linseed. On the top and the bottom of oilseed 
specimen two fast speed filter papers were respectively inserted for the purpose of preventing porous stones from blocking up with bits of broken oilseed. After the specimen was poured into the compression cell, the cell was mounted in a computer-controlled precision universal test machine. Initial thickness of specimen of peanut, soybean, sesame and linseed were measured, which were $33.3 \mathrm{~mm}, 28.2 \mathrm{~mm}, 29.8 \mathrm{~mm}$ and $27.7 \mathrm{~mm}$ respectively. Equal rate of applied pressure (0.1MPa.s-1) was used in the experiment. Four series of experiments were carried out under $18^{\circ} \mathrm{C}$ of room temperature and under double surface for flow of fluids through a porous stone. Each desired stress was 60MPa.

\subsection{Measured results and discussion}

Defined applied stress $\sigma$ and axial strain $\varepsilon$ are as follows

$$
\begin{gathered}
\sigma=\frac{F}{A} \\
\varepsilon=\frac{\Delta H}{H_{0}}
\end{gathered}
$$

Where: $F$ is the applied force acting on the specimen surface $(\mathrm{N}), A$ is the area of section of the specimen $\left(\mathrm{mm}^{2}\right), H_{0}$ is the initial height of the specimen $(\mathrm{mm})$, and $\Delta H$ is the displacement of the specimen $(\mathrm{mm})$.

Table 1 shows the measured results of strain with stress for extruded peanut, soybean, sesame and linseed compressed at thirteen stresses. Over 50 per cent strain for extruded soybean, sesame and linseed occurred at stress below 20MPa. Over 60 per cent strain for extruded peanut occurred at stress below 10MPa. No more than 13 per cent strain occurred at stress over $20 \mathrm{MPa}$ for extruded soybean sesame and linseed, and no more than 13 per cent strain occurred at stress over $10 \mathrm{MPa}$ for extruded peanut.

Prior to applying pressure, the specimen is a loose bed owing to a lots of pore space within oilseed specimen. After applying pressure on the specimen, pore space is rapidly dwindled due to gas vented rapidly and elastic deformation in the bed along with increasing pressing pressure. That is why the strains vary sharply at early stage for extruded oilseeds. The bed of oilseeds becomes dense due to plastic deformation. After that the bed becomes a fluid-solid coupling material owing to the cell wall of oilseed and granule broken. Last, the bed becomes oilseed cake as result of bond between broken oilseeds granule. The cake becomes denser and denser as oil is expelled. It explains the reason that no more than 13 per cent strain occurred at later stage for extruded oilseeds. 
Table 1. Variation of axial strain $\varepsilon(\%)$ with stress $\sigma(\mathrm{MPa})$ for the extruded peanut, soybean, sesame and linseed

\begin{tabular}{lccccccccccccc}
\hline \multirow{2}{*}{ oilseed } & \multicolumn{110}{c}{ Stress (MPa) } \\
\cline { 2 - 14 } & 0 & 5 & 10 & 15 & 20 & 25 & 30 & 35 & 40 & 45 & 50 & 55 & 60 \\
\hline peanut & 0 & 53.05 & 61.24 & 64.14 & 66.22 & 68.02 & 69.42 & 70.39 & 71.26 & 72.04 & 72.97 & 73.63 & 74.49 \\
soybean & 0 & 27.54 & 40.83 & 46.74 & 50.50 & 52.98 & 55.16 & 56.10 & 56.71 & 57.38 & 57.98 & 58.44 & 59.02 \\
sesame & 0 & 39.39 & 54.34 & 60.54 & 63.90 & 67.65 & 70.06 & 72.07 & 73.68 & 74.63 & 75.31 & 75.95 & 76.69 \\
linseed & 0 & 29.08 & 44.75 & 52.71 & 58.51 & 62.74 & 65.40 & 66.71 & 67.95 & 69.06 & 70.15 & 70.72 & 71.26 \\
\hline
\end{tabular}

\section{NEURAL NETWORKS IDENTIFICATION ALGORITHM}

It had been proved in theory that feed-forward neural networks trained with the back propagation (BP) can approximate continuous function and curve with arbitrary precision. The $\mathrm{BP}$ algorithm is a training learning process, which is divided into two processes, called forward-propagation and back-propagation respectively. Forward propagation is that input data from input layers are transmitted into hidden layer and into output layers after treated by hidden layers and output layers. If the practical output of neural networks is not expected output, the error between practical output and expected output will return through original path to change weights between layers, that is back-propagation. Forward propagation and back propagation are repeated until the prescribed error is met. The training learning process of artificial neural networks is actually one process of identification. So, BP neural network have been widely used in system identification to identify complex nonlinear system (Yang Jian et al., 2006; Sun Tao et al., 2005). The experiment indicated that stress-strain relationship for oilseeds during pressing was nonlinear. In this study, neural networks modeling techniques with BP network was used to predict the stress-strain relationship. Fig. 2 is the network model, which have $r$-inputs and one hidden layer.

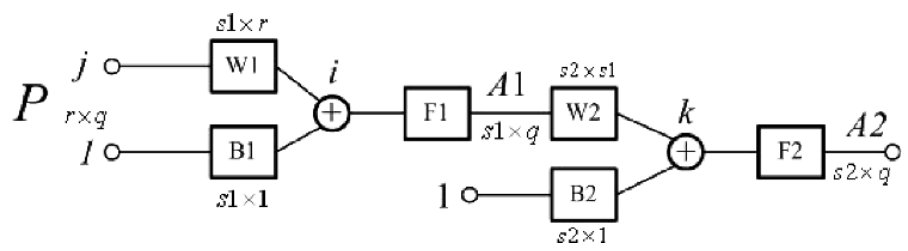

Fig. 2. Neural network model

$P$ is input matrix, $W 1$ is the weight matrix of the input layer, $B 1$ is the deviation matrix of the input layer, $F 1$ is the active function of the hidden layer, $A 1$ is the output matrix of the hidden layer, $W 2$ is the weight matrix of 
the output layer, $B 2$ is the deviation matrix of the output layer, $F 2$ is the active function of the output layer, and $A 2$ is the output matrix of the output layer.

\subsection{Forward transfer of information}

The $i$ th node output for hidden layer is

$$
a 1_{i}=f 1\left(\sum_{j=1}^{r} w 1_{i j} P_{j}+b 1_{i}\right), i=1,2, \cdots, s 1
$$

Where: $a 1_{i}$ is the $i$ th node output of the hidden layer, $f 1($.)is the active function of the hidden layer, $w 1_{i j}$ is the connection weight from the $j$ th input node to the $i$ th hidden node, $P_{j}$ is the $j$ th input, and $b 1_{i}$ is the $i$ th node bias value of the hidden layer.

The $k$ th node output for output layer is

$$
a 2_{k}=f 2\left(\sum_{i=1}^{s 1} w 2_{k i} a 1_{i}+b 2_{k}\right), k=1,2, \cdots, s 2
$$

Where: $a 2_{k}$ is the $k$ th node output of the output layer, $f 2($.$) is the active$ function of the out layer, $w 2_{k i}$ is the connection weight from the $i$ th output node of the hidden layer to the $k$ th output node of the output layer, and $b 2_{k}$ is the $k$ th node bias value of the output layer.

Adopting the error function as follows

$$
E(W, B)=\frac{1}{2} \sum_{k=1}^{s 2}\left(t_{k}-a 2_{k}\right)^{2}
$$

Where: $E(W, B)$ is the error function of the output, $t_{k}$ is the $k$ th node objective value of the output layer, and $a 2_{k}$ is the kth node output of the output layer.

\subsection{Change weight using gradient descent algorithm}

The weight from $i$ th input to $k$ th output is

$$
\begin{aligned}
\Delta w 2_{k i} & =-\eta \frac{\partial E}{\partial w 2_{k i}}=-\eta \frac{\partial E}{\partial a 2_{k}} \cdot \frac{\partial a 2_{k}}{\partial w 2_{k i}} \\
& =\eta\left(t_{k}-a 2_{k}\right) f 2^{\prime} a 1_{i}=\eta \delta_{k i} a 1_{i}
\end{aligned}
$$

Where: $\Delta w 2_{k i}$ is the change in weight of the output layer, $\eta$ is the learning rate, $f 2^{\prime}$ is the active function derivative of the output layer, $\delta_{k i}=\left(t_{k}-a 2_{k}\right) f 2^{\prime}=e_{k} f 2^{\prime}, e_{k}=t_{k}-a 2_{k}$, where $\delta_{k i}$ is the error from the $i$ th output node of the hidden layer to the $k$ th output node of the output layer, and $e_{k}$ is the $k$ th output error of the output layer. In the same way 


$$
\begin{aligned}
& \Delta b 2_{k i}=-\eta \frac{\partial E}{\partial b 2_{k i}}=-\eta \frac{\partial E}{\partial a 2_{k}} \cdot \frac{\partial a 2_{k}}{\partial b 2_{k i}} \\
& =\eta\left(t_{k}-a 2_{k}\right) f 2^{\prime}=\eta \delta_{k i}
\end{aligned}
$$

Where: $\Delta b 2_{k}$ is the change of the $k$ th node bias value of the output layer. The weight from $j$ th input to $i$ th output is

$$
\begin{aligned}
& \Delta w 1_{i j}=-\eta \frac{\partial E}{\partial w 1_{i j}}=-\eta \frac{\partial E}{\partial a 2_{k}} \cdot \frac{\partial a 2_{k}}{\partial a 1_{i}} \cdot \frac{\partial a 1_{i}}{\partial w 1_{i j}} \\
& =\eta \sum_{k=1}^{s 2}\left(t_{k}-a 2_{k}\right) f 2^{\prime} w 2_{k 1} f 1^{\prime} p_{j}=\eta \delta_{i j} p_{j}
\end{aligned}
$$

Where: $\Delta w 1_{i j}$ is the weight change of the hidden layer, $f 1^{\prime}$ is the active function derivative of the hidden layer, $\delta_{i j}=e_{i} f 1^{\prime}, e_{i}=\sum_{k=1}^{s 2} \delta_{k i} w 2_{k i}$, $\delta_{k i}=e_{k} f 2^{\prime}, e_{k}=t_{k}-a 2_{k}$, where: $\delta_{i j}$ is the error from the $j$ th input node of the input layer to the $i$ th output node of the hidden layer, and $e_{i}$ is the $i$ th node output error of the hidden layer. In the same way

$$
\Delta b 1_{i}=\eta \delta_{i j}
$$

Where: $\Delta b 1_{i}$ is the bias value change of the hidden layer.

A three layer feed-forward neural networks trained with the back propagation (BP) algorithm was adopted in this paper. Both input layer and output layer had one node, which represented applied pressures sequence and measured strains sequence respectively. Hidden layer had five nodes. 0.01 and 1000 were used as the error tolerance and the maximum number of training cycle respectively. Sigmoid function $f 1(s)=\left(1+e^{-s}\right)^{-1}$ was selected as active function $f 1(s)$. Linear function was selected as active function $f 2(s)$. The measured results had been taken as samples. 11 and 2 data were chosen randomly as training and testing sample respectively. The error function is

$$
E=\frac{1}{2} \sum_{k=1}^{11}\left(t_{k}-a 2_{k}\right)^{2}
$$

\subsection{Results and discussion}

Fig. 3 shows the curves of stress-strain predicted and measured, and Fig. 4 shows the curves of relationship between training times and error for peanut, soybean, sesame and linseed during the training process. The values of sum errors of the prediction for peanut, soybean, sesame and linseed were $0.00282,0.0083,0.0084$ and 0.0047 respectively. The training times were 11 , 


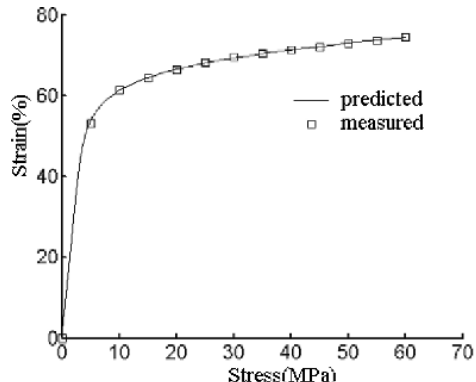

(a) peanut

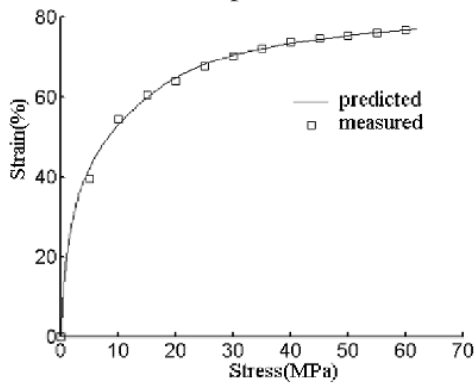

(c) sesame

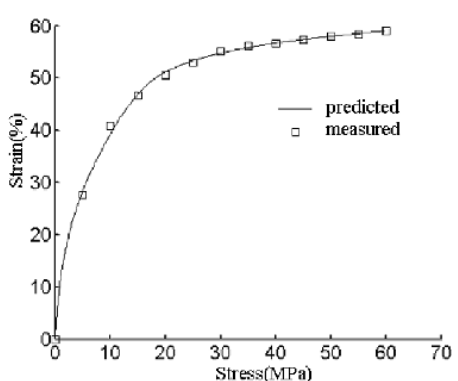

(b) soybean

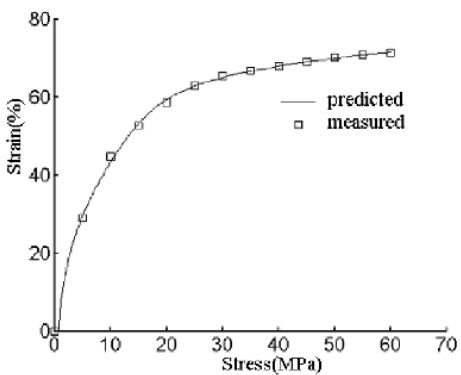

(d) linseed

Fig. 3. Comparison of prediction with measurement

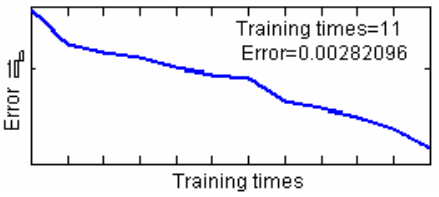

(a) peanut

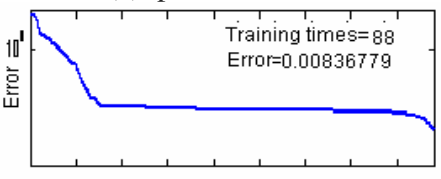

(c) sesame

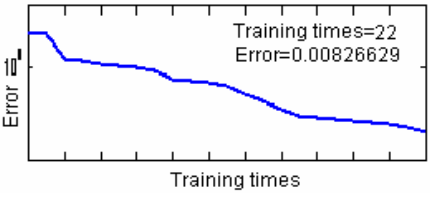

(b) soybean

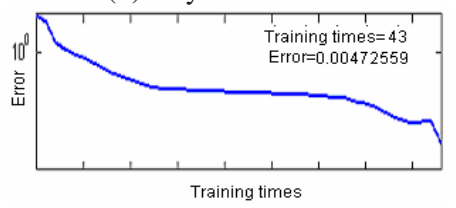

(d) linseed

Fig. 4. Relationship between training times and error

22,88 , and 43 respectively. It was found that there was a lack smooth for prediction curves for oilseeds due to over-fitting when the error tolerance is less than 0.001 .

\section{CONCLUSIONS}

Apparatus and procedures were developed to measure the stress-strain relationships for extruded peanut, soybean, sesame and linseed. Stress-strain 
relationships were measured compressed at twelve pressures $(5,10,15,20$, $25,30,35,40,45,50,55,60 \mathrm{MPa})$ under uniaxial cold pressing. The model was developed to predict the stress-strain relationship for extruded oilseeds based on BP neural network.

Results indicated that the stress-strain relationships were nonlinear. Over 50 per cent strains for extruded soybean, sesame and linseed occurred at stress below 20MPa. Over 60 per cent strain for extruded peanut occurred at stress below $10 \mathrm{MPa}$. No more than 13 per cent strain occurred at stress over $20 \mathrm{MPa}$ for extruded soybean sesame and linseed, and no more than 13 per cent strain occurred at stress over 10MPa for extruded peanut. There were significant increases in the values for the strains at early stage for extruded oilseeds, and there were no significant increases in the values for the strains at later stage for extruded oilseeds.

BP neural network can be used to predict the stress-strain relationship for oilseeds, which not only overcomes the difficulty for theoretical model development, but also avoids requiring considerable technique and experience for nonlinear regression analysis. No more than 0.0084 maximum error showed that the model predicted the stress-strain relationships with highly accuracy. In view of the predicted results and the simple model consisting of input and output layer with one node, and hidden layer with five nodes, the method of stress-strain prediction for oilseeds by using artificial neural networks is both feasible and effective.

\section{ACKNOWLEDGEMENTS}

Funding for this research was provided by Hubei Provincial Department of Education (P. R. China). The first author is grateful to the Wuhan Polytechnic University for providing him with pursuing a $\mathrm{PhD}$ degree at the Wuhan University of Technology.

\section{REFERENCES}

C. R. Sukumaran, B. P. N. Singh. Compression of bed of rapeseeds: the oil-point, Journal of Agricultural Engineering Research, 1989, 42:77-84

E. Davion, A. G. Meiering, F. J. Middendof. A theoretical stress model of rapeseed, Canadian Agricultural Engineering, 1979, 21(1):45-46

E. Davion, F. J. Middendof, W. K. Bilanski. Mechanical properties of rapeseed, Canadian Agricultural Engineering, 1975, 17(1):50-53

G. C. Mrema, P. B. Mcnulty. Mathematical Model of Mechanical Oil Expression from Oilseeds. Journal of Agricultural Engineering Research, 1985, 31:361-370 
H. J. Rasehom, H. D. Deicke, Xin Yaonian. Theory and praxis of decortication and cold pressing of rape seed, China oils and fats, 2000, 25(6): 50-54 (in Chinese)

P. C. Bargale, R. J. Ford, F. W. Sosulski, et al. J Irudayaj. Mechanical Oil Expression from Extruded Soybean Samples. Journal of the American oil chemists society, 1999, 76(2):223-229

P. C. Bargale, Jaswant Singh. Oil expression characteristics of rapeseed for a small capacity screw press, Journal of food Science Technolage, 2000, 37(2):130-134

P. C. Bargale, R. Ford, D. Jwulfsohn, et al. Measurement of consolidation and permeability properties of extruded soy under mechanical pressing, Journal of Agricultural Engineering Research, 1999, 74:155-165

Sun Tao, Cao Guangyi, Zhu Xinjian. Nonlinear modeling of PEMFC based on neural networks identification, Journal of Zhejiang University Science, Vol. 64, No. 5, 2005, 64(5):365-370

Yang Jian, Xu Bing, Yang Huayong. Noise identification for hydraulic axial piston pump based on artificial neural networks, Chinese Journal of mechanical engineering, 2006, 19(1):120-123

Zheng Xiao, Wan nong, Lin Guoxiang, et al. Research on microstructure of cold pressed cakes from decorticated rapeseed based on porosity, China Oils and Fats, 2004, 29(12):1417 (in Chinese)

Zheng Xiao, Zeng Shan, Lin Guoxiang, et al. Research on stress-strain of rapeseed and decorticated rapeseed by uniaxial cold pressing under single surface for flow of fluids through a porous medium, China oils and fats, 2004, 29(7):11-14 (in Chinese) 